\title{
Adubação orgânica na produção, rendimento e composição do óleo essen- cial da alfavaca quimiotipo eugenol
}

\author{
Luiz Antonio Biasi'; Eliana M Machado²; Ana Paula de J Kowalski²; Diana Signor²; Márcia A Alves²; \\ Francine I de Lima²; Cícero Deschamps'; Lílian C Côcco ${ }^{3}$; Agnes de Paula Scheer ${ }^{3}$ \\ ${ }^{1}$ UFPR-Dep ${ }^{\text {to }}$ de Fitotecnia e Fitossanitarismo, C. Postal 19061, 81531-990 Curitiba-PR; ${ }^{2}$ UFPR-Agronomia; ${ }^{3}$ UFPR/LACAUT-Dep ${ }^{\text {to }}$ de \\ Engenharia Química; biasi@ufpr.br
}

\section{RESUMO}

Este trabalho foi realizado na UFPR, município de Pinhais-PR, de outubro de 2005 a junho de 2006. Avaliou-se a resposta do Ocimum gratissimum à adubação orgânica com composto de esterco de carneiro, verificando a viabilidade dessa prática para o aumento no rendimento de biomassa, na quantidade e qualidade de seu óleo essencial. As mudas foram plantadas com espaçamento de $0,5 \mathrm{~m}$ entre plantas dentro das parcelas e $1 \mathrm{~m}$ entre as parcelas. Testaram-se 3 doses de adubação com composto orgânico (4; 8 e $\left.12 \mathrm{~kg} \mathrm{~m}^{-2}\right)$ e uma testemunha, com 5 repetições e 8 plantas por parcela. As colheitas foram realizadas aos 150 e 226 dias após o plantio, sendo avaliado o rendimento de massa fresca e massa seca total e de folhas, flores e caule, o teor de óleo essencial das folhas e flores e a sua composição por cromatógrafo a gás acoplado a um detector de massas (CG-MS). Houve diferença entre os tratamentos, quanto ao rendimento de biomassa na primeira colheita, onde o tratamento com $8 \mathrm{~kg} \mathrm{~m}^{-2}$ de composto orgânico foi superior à testemunha sem adubação, para a massa fresca de flores por planta e massa seca total e de flores por planta, mas não diferiu dos outros níveis de adubação. Na segunda colheita não houve diferença entre os tratamentos. Quanto ao rendimento de óleo essencial não houve diferença entre os tratamentos nas duas colheitas. Também não foram observadas grandes variações nos componentes do óleo essencial decorrente dos tratamentos aplicados. Entretanto, ocorreram variações na composição do óleo essencial quando extraído das folhas e das flores, sendo o teor médio de eugenol de $90,4 \%$ nas folhas e $80,8 \%$ nas flores, na colheita em março. Já na colheita em junho, houve uma redução do teor de eugenol nas folhas e uma elevação dos teores, principalmente de alfa trans trans farneseno, beta bisaboleno, beta cariofileno, germacrene D e alfa selineno.

Palavras-chave: Ocimum gratissimum, composto orgânico, produtividade.

\begin{abstract}
Organic fertilization in the production, yield and chemical composition of basil chemotype eugenol

This work was carried out at the Universidade Federal do Parana, Paraná State, Brazil, from October 2005 to June 2006. The effect of organic fertilization with sheep manure was evaluated on Ocimum gratissimum development and the viability of this practice was determined to increase biomass and essential oil yield and quality. The plants were spaced $0.5 \mathrm{~m}$ from each other in the experimental units which were separated in $1 \mathrm{~m}$. Three rates of organic fertlization (4; 8 and $12 \mathrm{~kg} \mathrm{~m}^{-2}$ ) were compared to control (without fertilizers), with 5 replications and 8 plants by experimental unit. Plants were harvested 150 and 226 days after planting, and the total, leaves, flowers and stems fresh and dried biomass, essential oil yield from leaves and flowers and composition by GC/MS beign evaluated. There was significant difference among the treatments on biomass production at the first harvest, where the plants treated with $8 \mathrm{~kg} \mathrm{~m}^{-}$ ${ }^{2}$ of organic fertilizer presented higher flowers fresh biomass, and flowers and total dry biomass than plants without fertilizer (control plants), but with no difference compared to other organic fertilization levels. At the second harvest there were no differences among treatments. Regarding the essential oil yield no differences were found comparing the organic fertilization levels in both harvest times. In addition, the essential oil composition was similar on plants from all treatments. However, some variations in composition were observed when the essential oil was extracted from flowers and leaves, where the eugenol percentage was $90.4 \%$ on leaves and $80.8 \%$ on flowers at the first harvest. At the second harvest, a reduction of eugenol content and an increase of alpha trans trans farnesene, beta bisabolene, beta cariophilene, germacrene D and alfa selineno was found.
\end{abstract}

Keywords: Ocimum gratissimum, organic compost, yield.

(Recebido para publicação em 17 de outubro de 2007; aceito em 19 de fevereiro de 2009) (Received on October 17, 2007; accapted on February 19, 2009)

$\mathrm{O}$ Ocimum gratissimum, também conhecido como alfavaca-cravo ou alfavacão, é um subarbusto aromático, com até um metro de altura, originário do Oriente e subespontâneo em todo o Brasil. Possui folhas ovaladolanceoladas, de bordos duplamente dentados, membranáceas, com 4 a $8 \mathrm{~cm}$ de comprimento. As flores são pequenas, roxo-pálidas, dispostas em racemos paniculados eretos e geralmente em grupos de três (Lorenzi \& Matos, 2002).
Existem vários quimiotipos, como ocorre em outras espécies do mesmo gênero, sendo um quimiotipo muito comum o eugenol, que possui essa substância em maior quantidade na composição do óleo essencial. O eugenol é a substância característica do aroma do cravo-da-índia. No nordeste do Brasil, encontra-se um quimiotipo de $O$. gratissimum que apresenta ação diurética, denominado quiôio (Blank et al., 2003), enquanto o quimiotipo eugenol apresenta ação anti-séptica (Matos et al., 2000). No óleo essencial extraído de $O$. gratissimum foram encontrados os seguintes compostos: 1,8 cineol, eugenol, metil-eugenol, timol, pcimeno, cis-ocimeno e cis-cariofileno, sendo que o eugenol foi indicado como responsável por inibir o crescimento de Staphylococcus aureus, Shigella flexneri, Salmonella enteritidis e Escherichia coli, Klebsiella sp. e Proteus mirabilis (Nakamura et al., 1999). 
Ao analisar a atividade antibacteriana de óleos essenciais de plantas medicinais, frente a 100 cepas de bactérias isoladas de indivíduos da comunidade com diagnóstico de infecção urinária, foi verificado que, embora menos eficiente que Salvia officinalis, o O. gratissimum apresentou atividade antimicrobiana em $16 \%$ das cepas estudadas, exceto Klebsiella oxytoca e Pseudomonas aeruginosa (Pereira et al., 2004).

O óleo essencial desta planta apresenta atividade antifúngica comprovada contra os fungos fitopatogênicos Botryosphaeria rhodina e duas espécies de Alternaria sp. O eugenol apresenta atividade contra Alternaria isolada de tomate $\left(\mathrm{A}_{1}\right)$ e Penicillium chrysogenum (Faria et al., 2006). Também foi atribuído ao eugenol a atividade contra os fungos Penicillium chrysogenum, Rhizoctonia sp., Aspergillus niger e duas espécies de Alternaria sp. isoladas de tomate e cenoura (Rosset et al., 2005).

Além da atividade antifúngica e antibacteriana, também foi observada a ação larvicida do óleo essencial dessa espécie contra Aedes aegypti, o mosquito transmissor da dengue, cuja dose letal média foi de $60 \mathrm{ppm}$ (Cavalcanti et al., 2004) e ação repelente do óleo essencial contra Simulium damnosum, o transmissor de Onchocerca volvulus na Nigéria. A aplicação tópica na concentração de $20 \%$ reduziu a taxa de picadas em até $90,2 \%$ e protegeu por 3 horas (Usip et al., 2006).

O metabolismo primário e secundário é afetado por diversos fatores externos, sendo a disponibilidade de nutrientes um desses fatores. A influência da adubação, no desenvolvimento e produção de óleos essenciais, tem sido demonstrada em diversas plantas medicinais. O manjericão (Ocimum basilicum L.) cv. Genovese apresentou maior produção de matéria seca da parte aérea e maior rendimento de óleo essencial quando foi adubado com um adubo químico formulado com macro e micronutrientes e esterco de galinha (Blank et al., 2005). Para o capim-limão (Cymbopogon citratus), a adubação mineral com NPK e esterco bovino também resultou em maior rendimento de biomassa seca e teor de óleo essencial, quando as folhas foram colhidas às 07:00 hs (Silva et al., 2003). Entretanto, nem sempre a adubação apresenta resposta positiva, como foi observado para Lippia alba, onde a adubação orgânica não afetou a produção de matéria seca foliar nem o teor de óleo essencial (Santos \& Innecco, 2004). Para o chambá (Justicia pectoralis var. stenophylla), as adubações orgânicas e minerais não influenciaram o crescimento da planta e a produção de biomassa e o teor de óleo essencial apresentou uma tendência de decréscimo com o incremento nas doses de esterco bovino em cada uma das formulações de NPK (Bezerra et al., 2006).

O objetivo desse trabalho foi avaliar a resposta do $O$. gratissimum à adubação orgânica, verificando a viabilidade dessa prática para o aumento no rendimento de biomassa, quantidade e qualidade de seu óleo essencial.

\section{MATERIAL E MÉTODOS}

$\mathrm{O}$ experimento na Universidade $\mathrm{Fe}$ deral do Paraná, localizada no município de Pinhais no Paraná (2525' S; 4042' W; altitude média de $930 \mathrm{~m}$ ). O solo em análise prévia possuía: $\mathrm{pH} \mathrm{SMP}=6,6 ; \mathrm{Al}^{+3}=$ $0,0 \mathrm{cmol}_{\mathrm{c}} \mathrm{dm}^{-3} ; \mathrm{H}^{+}+\mathrm{Al}^{+3}=3,2 \mathrm{cmol}_{\mathrm{c}} \mathrm{dm}^{-3}$; $\mathrm{Ca}^{+2}=9,6 \mathrm{cmol}_{\mathrm{c}} \mathrm{dm}^{-3} ; \mathrm{Mg}^{+2}=5,5 \mathrm{cmol}_{\mathrm{c}} \mathrm{dm}^{-3}$; $\mathrm{K}^{+}=1,59 \mathrm{cmol}_{\mathrm{c}} \mathrm{dm}^{-3} ; \mathrm{P}=159 \mathrm{mg} \mathrm{dm}^{-3} ; \mathrm{C}=$ $36,5 \mathrm{~g} \mathrm{dm}^{-3}$; saturação por bases $=84 \% \mathrm{e}$ teor de argila de $400 \mathrm{~g} \cdot \mathrm{kg}^{-1}$.

As mudas produzidas a partir de sementes, em bandejas de poliestireno expandido de 128 células, com 2 a 3 folhas, foram plantadas em 31/10/05. Utilizouse como adubo orgânico um composto à base de esterco curtido de carneiro $\mathrm{e}$ palha seca (produzido em julho/05), com teor aproximado de umidade de $44 \%$. O composto analisado possuia $\mathrm{pH} \mathrm{SMP}=$ 6,$9 ; \mathrm{Al}^{+3}=0,0 \mathrm{cmol}_{\mathrm{c}} \mathrm{dm}^{-3} ; \mathrm{H}^{+}+\mathrm{Al}^{+3}=2,5$ $\mathrm{cmol}_{\mathrm{c}} \mathrm{dm}^{-3} ; \mathrm{Ca}^{+2}=10,8 \mathrm{cmol}_{\mathrm{c}} \mathrm{dm}^{-3} ; \mathrm{Mg}^{+2}=$ $10,4 \mathrm{cmol}_{\mathrm{c}} \mathrm{dm}^{-3} ; \mathrm{K}^{+}=3,4 \mathrm{cmol}_{\mathrm{c}} \mathrm{dm}^{-3} ; \mathrm{P}=$ $728 \mathrm{mg} \mathrm{dm}^{-3}$; saturação por bases $=91 \%$.

O experimento foi instalado com delineamento em blocos ao acaso, com 4 tratamentos, 5 repetições e 8 plantas em cada parcela. $\mathrm{O}$ espaçamento adotado foi de $0,5 \mathrm{~m}$ entre plantas e $1,0 \mathrm{~m}$ entre parcelas. Os tratamentos aplicados foram testemunha (sem adubação orgânica) e 4; 8 e 12 $\mathrm{kg} \mathrm{m}^{-2}$ de composto parcelado em duas aplicações em fevereiro e abril/06.
A primeira colheita foi realizada de 21 a 23/03/06, sendo analisadas as características: massa fresca e seca total, de folhas, flores e caule por planta e teor de óleo essencial nas folhas e flores. $\mathrm{Na}$ segunda colheita, realizada em 07/06/06, foram avaliadas as mesmas características, apenas, devido ao menor crescimento das plantas, a massa das folhas e caules foram pesadas juntas. A produtividade de óleo essencial em $\mathrm{L} \mathrm{ha}^{-1}$ foi estimada para uma população de 16.000 plantas ha $^{-1}$. A extração de óleo essencial foi realizada no mesmo dia de cada colheita, sendo feita pelo método de hidrodestilação com $100 \mathrm{~g}$ de massa fresca de folhas e flores em $1000 \mathrm{~mL}$ de água destilada, com destilador tipo Clevenger, permanecendo por um período de 3 horas. Para estimar o teor de óleo essencial em relação à matéria seca, foi determinada a massa seca de uma amostra de $10 \mathrm{~g}$ do material destilado.

O óleo essencial obtido foi analisado em cromatógrafo a gás acoplado a espectrofotômetro de massa (CG-MS) no Laboratório de Análise de Combustíveis Automotivos (LACAUT) da UFPR. Para identificação dos componentes do óleo essencial, utilizou-se o equipamento Varian, modelo CP3800/Saturno 2000, com íon trap e coluna capilar Chrompack de sílica fundida (CP-SIL 8 CB, 0,25 mm de diâmetro interno, $30 \mathrm{~m}$ de comprimento e $0,25 \mathrm{~mm}$ de filme líquido). Uma quantidade de $0,2 \mu \mathrm{L}$ de amostra foi injetada a $250^{\circ} \mathrm{C}$ (split 1:300), com uma vazão

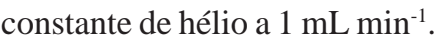

$\mathrm{O}$ espectro de massa de cada componente do óleo essencial foi comparado aos espectros do acervo das bibliotecas Saturn (GC-MS versão 5.51) e Nist (98 MS, versão 1.7). Confirmou-se a identificação pelo cálculo do índice de retenção de cada componente (Adams, 1995), utilizando-se uma série homóloga de hidrocarbonetos (C5 - C20) como picos de referência.

Para a quantificação dos constituintes, utilizou-se um cromatógrafo gasoso Varian, modelo CP-3800, com detector FID (CG-FID). Para a separação cromatográfica, utilizou-se uma coluna capilar Chrompack, de silica fundida CPSIL 8 CB, 0,25 mm de diâmetro interno, $30 \mathrm{~m}$ de comprimento e $0,25 \mu \mathrm{m}$ de filme líquido. As condições de análise para 
Tabela 1. Massa fresca e seca total, de folhas, de flores e de caule $\left(\mathrm{g}\right.$ planta $\left.^{-1}\right)$ em alfavaca quimiotipo eugenol em resposta a doses de adubação orgânica na primeira colheita de 21-23/03/06 (total, leaves, flowers and stems fresh and dried biomass of basil chemiotype eugenol at the first harvest from March $21^{\text {st }}$ to $23^{\text {th }}$, 2006). Pinhais, UFPR, 2006.

\begin{tabular}{lcccccccc}
\hline Tratamento & $\begin{array}{c}\text { Massa fresca } \\
\text { total }\end{array}$ & $\begin{array}{c}\text { Massa fresca } \\
\text { de folhas }\end{array}$ & de flores & de caules & $\begin{array}{c}\text { Massa seca } \\
\text { total }\end{array}$ & $\begin{array}{c}\text { Massa seca } \\
\text { de folhas }\end{array}$ & $\begin{array}{c}\text { Massa seca } \\
\text { de flores }\end{array}$ & $\begin{array}{c}\text { Massa seca } \\
\text { de caules }\end{array}$ \\
\hline Testemunha & $546,94 \mathrm{~b}^{1}$ & $270,78 \mathrm{a}$ & $100,86 \mathrm{~b}$ & $175,26 \mathrm{~b}$ & $116,72 \mathrm{~b}$ & $50,28 \mathrm{a}$ & $20,30 \mathrm{~b}$ & $46,12 \mathrm{a}$ \\
$4 \mathrm{~kg} \mathrm{~m}^{-2}$ & $689,90 \mathrm{a}$ & $313,66 \mathrm{a}$ & $138,34 \mathrm{ab}$ & $237,90 \mathrm{a}$ & $154,38 \mathrm{ab}$ & $65,26 \mathrm{a}$ & $29,10 \mathrm{ab}$ & $59,98 \mathrm{a}$ \\
$8 \mathrm{~kg} \mathrm{~m}^{-2}$ & $700,56 \mathrm{a}$ & $300,82 \mathrm{a}$ & $168,06 \mathrm{a}$ & $231,66 \mathrm{a}$ & $166,46 \mathrm{a}$ & $59,88 \mathrm{a}$ & $37,82 \mathrm{a}$ & $68,74 \mathrm{a}$ \\
$12 \mathrm{~kg} \mathrm{~m}^{-2}$ & $638,24 \mathrm{ab}$ & $302,54 \mathrm{a}$ & $124,70 \mathrm{ab}$ & $210,96 \mathrm{ab}$ & $136,94 \mathrm{ab}$ & $56,28 \mathrm{a}$ & $25,20 \mathrm{ab}$ & $55,40 \mathrm{a}$ \\
\hline $\mathrm{CV}(\%)$ & 11,8 & 14,9 & 25,3 & 15,2 & 20,1 & 18,2 & 32,7 & 27,8 \\
\hline
\end{tabular}

${ }^{1}$ Médias seguidas pela mesma letra não diferem pelo teste de Duncan a $5 \%$ de probabilidade (means followed by the same letter do not differ by Duncan test at $5 \%$ of probability).

Tabela 2. Massa fresca e seca total, de caules e folhas e de flores de alfavaca quimiotipo eugenol e rendimento e produtividade de óleo essencial de folhas em resposta a níveis de adubação orgânica na segunda colheita em 07/06/06 (total, stems and leaves, and flowers fresh and dried biomass of basil chemiotype eugenol and essential oil yield and productivity in leaves with organic fertlization levels at the second harvest on June $\left.7^{\text {th }}, 2006\right)$. Pinhais, UFPR, 2006.

\begin{tabular}{|c|c|c|c|c|c|c|c|c|}
\hline Tratamentos & $\begin{array}{c}\text { Massa fresca } \\
\text { total } \\
\left(\text { g planta }^{-1}\right)\end{array}$ & $\begin{array}{c}\text { Massa fresca } \\
\text { de caules e } \\
\text { folhas } \\
\left.\text { (g planta }^{-1}\right)\end{array}$ & $\begin{array}{c}\text { Massa fresca } \\
\text { de flores } \\
\left(\text { g planta }^{-1}\right)^{1}\end{array}$ & $\begin{array}{c}\text { Massa seca } \\
\text { total } \\
\left(g_{\text {planta }}^{-1}\right)\end{array}$ & $\begin{array}{c}\text { Massa seca } \\
\text { de caules e } \\
\text { folhas } \\
\left(\text { g planta }^{-1}\right)\end{array}$ & $\begin{array}{c}\text { Massa seca } \\
\text { de flores } \\
\left(\mathrm{g} \mathrm{planta}^{-1}\right)^{1}\end{array}$ & $\begin{array}{c}\text { Óleo } \\
\text { essencial de } \\
\text { folha }^{\mathrm{s}} \\
\left(\mu \mathrm{L} \mathrm{g}^{-1} \mathrm{massa}\right. \\
\text { seca })^{1}\end{array}$ & $\begin{array}{c}\text { Óleo } \\
\text { essencial de } \\
\text { folhas } \\
\left(\mathrm{L} \mathrm{ha}^{-1}\right)^{1}\end{array}$ \\
\hline Testemunha & $196,78 a^{2}$ & $188,60 \mathrm{a}$ & $8,18 \mathrm{a}$ & 29,59 a & $27,78 \mathrm{a}$ & $1,80 \mathrm{a}$ & $9,59 a$ & $4,13 a$ \\
\hline $4 \mathrm{~kg} \mathrm{~m}^{-2}$ & $170,44 \mathrm{a}$ & 165,66 a & $4,78 \mathrm{a}$ & $25,22 \mathrm{a}$ & $24,38 \mathrm{a}$ & $0,84 a$ & $12,54 \mathrm{a}$ & $4,84 \mathrm{a}$ \\
\hline $8 \mathrm{~kg} \mathrm{~m}^{-2}$ & $176,25 \mathrm{a}$ & 168,30 a & $7,94 \mathrm{a}$ & $27,82 \mathrm{a}$ & 26,19 a & $1,61 \mathrm{a}$ & $10,27 \mathrm{a}$ & $4,31 \mathrm{a}$ \\
\hline $12 \mathrm{~kg} \mathrm{~m}^{-2}$ & $168,70 \mathrm{a}$ & $163,02 \mathrm{a}$ & 5,68 a & 24,47 a & $23,40 \mathrm{a}$ & $1,07 \mathrm{a}$ & $7,74 \mathrm{a}$ & $2,88 \mathrm{a}$ \\
\hline$\overline{C V}(\%)$ & 17,1 & 15,1 & 50,33 & 25,3 & 22,9 & 32,2 & 29,4 & 24,0 \\
\hline
\end{tabular}

${ }^{1}$ Os dados originais foram transformados em raiz de $(\mathrm{x}+1)$ para análise estatística (the original data were transformed on root of $(\mathrm{x}+1)$ for statistical analysis); ${ }^{2}$ Médias seguidas pela mesma letra não diferem pelo teste de Duncan a 5\% de probabilidade (means followed by the same letter do not differ by Duncan test at $5 \%$ of probability).

esta coluna foram: a) temperatura do injetor: $250^{\circ} \mathrm{C}$, split $1: 300$; b) quantidade de amostra injetada: $1,0 \mu \mathrm{L} ; \mathrm{c})$ gás de arraste: hélio a $1,0 \mathrm{~mL} \mathrm{~min}^{-1} ; \mathrm{d}$ ) temperatura do detector: $300^{\circ} \mathrm{C}$; e) programação de temperatura do forno: temperatura inicial de $60^{\circ} \mathrm{C}$, elevação de temperatura a $140^{\circ} \mathrm{C}$ na razão de $3^{\circ} \mathrm{C} \cdot \mathrm{min}^{-1}$; elevação de temperatura a $240^{\circ} \mathrm{C}$ na razão de $30^{\circ} \mathrm{C}$ $\mathrm{min}^{-1}$, permanecendo por 5 minutos; f) tempo total de corrida: 35 minutos.

Os dados obtidos foram submetidos à análise de variância e as médias dos tratamentos comparadas pelo teste de Duncan com 5\% de significância.

\section{RESULTADOS E DISCUSSÃO}

Devido ao grande desenvolvimento observado na primeira colheita, optouse por separar os caules das folhas, já que os caules encontravam-se muito lignificados e representavam $40 \%$ da massa seca total. Observou-se na primeira colheita, que as três doses aplica- das de composto orgânico não diferiram entre si com relação à biomassa fresca e seca produzida. Apenas a dose de 8 $\mathrm{kg} \mathrm{m}^{-2}$ foi superior à testemunha quanto à massa fresca de flores e massa seca total e de flores (Tabela 1). A massa fresca e seca de folhas não diferiu entre os tratamentos, da mesma forma como já foi observado por Chaves et al. (2002) para a massa seca de folhas de $O$. gratissimum, utilizando as mesmas doses de adubação com esterco de galinha. Silva et al. (2003) verificaram que a adubação orgânica com $4 \mathrm{~L} \mathrm{~m}^{-2}$ de esterco bovino apresentou rendimento de biomassa seca inferior à adubação orgânica mais mineral com nitrogênio, fósforo e potássio para o capim-limão (Cymbopogon citratus). O manjericão (Ocimum basilicum) cv. Genovese também apresentou menor rendimento de biomassa seca quando foi adubado apenas com esterco bovino $\left(1,8 \mathrm{~kg} \mathrm{~m}^{-2}\right) \mathrm{em}$ comparação com adubação mineral com macro e micronutrientes sozinha ou combinada com esterco de galinha ou bovino (Blank et al., 2005).

Na segunda colheita não houve diferença entre os tratamentos, para todas as variáveis de biomassa analisadas (Tabela 2). Entretanto, foi observada uma redução no desenvolvimento das plantas em relação à primeira colheita. Nessa época, que corresponde ao final do outono, é comum uma redução da temperatura média diária, que deve ter causado uma redução na taxa de crescimento das plantas, pois já foi demonstrado para $O$. gratissimum var. macrophyllum, que médias térmicas semanais abaixo de $80^{\circ} \mathrm{C}$ dia $^{-1}$ são limitantes para o seu crescimento (Costa Filho et al., 2006). Nessa colheita, a massa seca total média foi de $26,8 \mathrm{~g}$ por planta, enquanto na primeira colheita foi de 143,6 g por planta. O florescimento também foi bastante reduzido na segunda colheita, onde a massa seca média de flores foi de apenas $1,3 \mathrm{~g}$ por planta, enquanto na primeira colheita foi de 28,1 
Tabela 3. Rendimento e produtividade de óleo essencial em folhas e flores de alfavaca quimiotipo eugenol em resposta a doses de adubação orgânica na primeira colheita de 21-23/03/06 (essential oil yield and producitivity in basil chemiotype eugenol leaves and flowers with organic fertlization levels at the first harvest from March $21^{\text {st }}$ to $23^{\text {th }}, 2006$ ). Pinhais, UFPR, 2006.

\begin{tabular}{|c|c|c|c|c|}
\hline Tratamento & $\begin{array}{c}\text { Óleo essencial } \\
\text { folha }\left(\mu \mathrm{L} \mathrm{g}^{-1}\right. \\
\text { massa seca) }{ }^{1}\end{array}$ & $\begin{array}{c}\text { Óleo essencial } \\
\text { flor }\left(\mu \mathrm{L} \mathrm{g}^{-1}\right. \\
\text { massa seca) } 1\end{array}$ & $\begin{array}{c}\text { Óleo essencial } \\
\text { folha } \\
\left(\mathrm{L} \mathrm{ha}^{-1}\right)^{1}\end{array}$ & $\begin{array}{c}\text { Óleo essencial } \\
\text { flor } \\
\left(\mathrm{L} \mathrm{ha}^{-1}\right)^{1}\end{array}$ \\
\hline Testemunha & $19,42 a^{2}$ & $8,10 a$ & $15,15 a$ & $2,60 \mathrm{a}$ \\
\hline $4 \mathrm{~kg} \mathrm{~m}^{-2}$ & $17,91 \mathrm{a}$ & $9,43 \mathrm{a}$ & $17,44 \mathrm{a}$ & $4,91 \mathrm{a}$ \\
\hline $8 \mathrm{~kg} \mathrm{~m}^{-2}$ & $13,55 \mathrm{a}$ & $6,80 \mathrm{a}$ & $13,00 \mathrm{a}$ & $4,10 \mathrm{a}$ \\
\hline $12 \mathrm{~kg} \mathrm{~m}^{-2}$ & $17,92 \mathrm{a}$ & $9,43 \mathrm{a}$ & $15,96 \mathrm{a}$ & $3,61 \mathrm{a}$ \\
\hline$\overline{C V}(\%)$ & 15,4 & 22,1 & 21,1 & 24,0 \\
\hline
\end{tabular}

${ }^{1}$ Os dados originais foram transformados em raiz de $(x+1)$ para análise estatística (the original data were transformed to root of $(\mathrm{x}+1)$ for statistical analysis); ${ }^{2}$ Médias seguidas pela mesma letra não diferem pelo teste de Duncan a $5 \%$ de probabilidade (means followed by the same letter do not differ by Duncan test at $5 \%$ of probability).

g por planta. Num experimento com $O$. gratissimum em Botucatu (SP), foi observado maior rendimento de folhas na colheita realizada no inverno (agosto), em relação à colheita de outono (maio) (Chaves et al., 2002). Entretanto, as condições climáticas diferem bastante entre esses locais, pois em Pinhais (PR), onde foi realizado o presente experimento, as geadas durante o inverno danificaram toda a parte aérea das plantas, impedindo a continuidade do experimento.

O rendimento de óleo essencial não diferiu entre os tratamentos nas duas épocas de colheita (Tabelas 2 e 3), observando-se para as folhas em média $17,2 \mu \mathrm{L} \mathrm{g}^{-1}$ de massa seca $(1,72 \%)$ na primeira colheita e $10,0 \mu \mathrm{L} \mathrm{g}^{-1}$ massa seca (1\%) na segunda colheita. Redução no rendimento de óleo essencial semelhante também ocorreu no trabalho de Chaves et al. (2002), de 1,29\% para 1,08\%, sendo atribuída a menor temperatura ocorrida no período da segunda colheita. As inflorescências também apresentaram óleo essencial, porém com praticamente metade do rendimento das folhas, em média $8,4 \mu \mathrm{L} \mathrm{g}^{-1}$ massa seca $(0,84 \%)$. Em escala comercial onde a planta inteira é destilada, as inflorescências contribuirão para aumentar o rendimento de óleo essencial, que na primeira colheita foi estimado em 15,4 $\mathrm{L} \mathrm{ha}^{-1}$ para as folhas e 3,8 $\mathrm{L} \mathrm{ha}^{-1}$ para as flores.

Ao analisar a composição química do óleo essencial, foi confirmado que o quimiotipo estudado de $O$. gratissimum foi o eugenol, pois essa substância foi encontrada em quantidades muito superiores às demais (Tabela 4). Não foram encontradas diferenças marcantes na composição do óleo essencial devido aos níveis de adubação estudados, mas ocorreram mudanças na composição com relação à época de colheita e origem do material destilado, de folhas ou de flores. Na primeira colheita foi encontrado em média 90,4\% de eugenol nas folhas, enquanto na segunda colheita 0 teor caiu para $65,4 \%$, o que resultou no aumento da quantidade dos demais compostos, principalmente alfa trans trans farneseno, beta bisaboleno, beta cariofileno, germacrene D e alfa selineno. As flores também apresentaram variação na proporção dos compostos, sendo menor o teor de eugenol, em média $80,8 \%$ e maiores os teores de alfa trans trans farneseno, beta bisaboleno, beta cariofileno, germacrene D e alfa selineno (Tabela 4). Os teores de eugenol obtidos no presente trabalho foram superiores aos obtidos por Chaves et al. (2002), que encontraram no máximo $47,29 \%$ de eugenol na colheita de outono, valor que foi superior ao obtido no inverno $(33,23 \%)$. Também foram superiores aos encontrados por Jirovetz et al. (2003) para O. gratissimum cultivado no sul da Índia, com $63,36 \%$ de eugenol. Esses autores também encontraram 9,11\% de (Z)-B-ocimene, $8,84 \%$ de germacrene $\mathrm{De} 3,89 \%$ de $\beta$-cariofileno. Silva et al. (1999) ao analisarem a variação do teor de eugenol no óleo essen-

Tabela 4. Composição química em porcentagem do óleo essencial de folhas e flores de alfavaca quimiotipo eugenol em resposta a doses de adubação orgânica em $\mathrm{kg} \mathrm{m}^{2}$ na primeira colheita de 21-23/03 e segunda colheita de 07/06/06 (chemical composition in percentage of essential oil from leaves and flowers of basil chemiotype eugenol with organic fertilization levels $\mathrm{kg} \mathrm{m}^{2}$ at the first harvest from March $21^{\text {st }}$ to $23^{\text {th }}$ and second harvest at June $7^{\text {th }}$, 2006). Pinhais, UFPR, 2006.

\begin{tabular}{|c|c|c|c|c|c|c|c|c|c|c|c|c|c|}
\hline \multirow{3}{*}{ Composto } & \multirow{3}{*}{$\mathbf{I R}^{\mathbf{1}}$} & \multicolumn{8}{|c|}{$1^{\text {a }}$ Colheita } & \multirow{2}{*}{\multicolumn{4}{|c|}{$\begin{array}{l}2^{\mathrm{a}} \text { Colheita } \\
\text { Folha }\left(\mathrm{kg} \mathrm{m}^{-2}\right) \\
\end{array}$}} \\
\hline & & \multicolumn{4}{|c|}{ Folha $\left(\mathrm{kg} \mathrm{m}^{-2}\right)$} & \multicolumn{4}{|c|}{ Flor $\left(\mathrm{kg} \mathrm{m}^{-2}\right)$} & & & & \\
\hline & & 0 & 4 & 8 & 12 & 0 & 4 & 8 & 12 & 0 & 4 & 8 & 12 \\
\hline Eugenol & 1359 & 90,50 & 90,44 & 90,26 & 90,53 & 81,22 & 86,14 & 76,40 & 79,56 & 62,12 & 72,92 & 65,71 & 61,01 \\
\hline Alfa trans trans farneseno & 1503 & 3,85 & 3,93 & 4,13 & 3,92 & 8,35 & 5,61 & 11,04 & 9,25 & 15,08 & 9,89 & 13,84 & 12,84 \\
\hline Beta bisaboleno & 1507 & 1,87 & 1,93 & 1,90 & 1,79 & 3,83 & 2,57 & 5,14 & 4,11 & 5,93 & 6,14 & 7,32 & 7,86 \\
\hline Beta cariofileno & 1418 & 0,66 & 0,66 & 0,64 & 0,64 & 1,03 & 0,73 & 1,24 & 1,08 & 2,35 & 1,71 & 2,25 & 2,10 \\
\hline Germacrene D & 1480 & 0,54 & 0,57 & 0,58 & 0,58 & 1,31 & 1,01 & 1,57 & 1,38 & 3,77 & 2,63 & 3,37 & 3,08 \\
\hline Alfa selineno & 1494 & 0,17 & 0,18 & 0,18 & 0,17 & 0,33 & 0,27 & 0,31 & 0,30 & 0,94 & 0,69 & 0,91 & 0,74 \\
\hline Beta bourboneno & 1382 & 0,05 & 0,05 & 0,04 & 0,04 & 0,06 & 0,04 & 0,07 & 0,06 & 0,14 & 0,11 & 0,14 & 0,13 \\
\hline Beta cubebeno & 1386 & 0,04 & 0,03 & 0,03 & 0,04 & 0,09 & 0,07 & 0,11 & 0,09 & 0,37 & 0,28 & 0,35 & 0,26 \\
\hline Linalol & 1099 & 0,14 & 0,16 & 0,15 & 0,18 & 0,58 & 0,58 & 0,54 & 0,51 & 0,37 & 0,65 & 0,63 & 0,50 \\
\hline Isoledeno & 1374 & 0,06 & 0,06 & 0,06 & 0,07 & 0,16 & 0,11 & 0,21 & 0,16 & 0,61 & 0,43 & 0,58 & 0,50 \\
\hline Delta cadideno & 1521 & 0,03 & 0,03 & 0,03 & 0,03 & 0,07 & 0,05 & 0,10 & 0,09 & 0,27 & 0,18 & 0,24 & 0,24 \\
\hline
\end{tabular}

${ }^{1}$ Índice de retenção (Retention Index) 
cial de folhas de $O$. gratissimum ao longo do dia, observaram ao meio dia o maior rendimento, que atingiu $98 \%$.

Conclui-se que a alfavaca-cravo responde pouco à adubação orgânica e o pequeno aumento de biomassa não corresponde a um aumento no rendimento de óleo essencial. O teor de óleo essencial e o teor de eugenol são maiores nas folhas do que nas flores.

\section{AGRADECIMENTOS}

Os autores agradecem à Secretaria de Estado de Ciência e Tecnologia (SETI)/Governo do Estado do Paraná pelo apoio financeiro e concessão de bolsa para as alunas de graduação e ao CNPq pela bolsa de Produtividade em Pesquisa do primeiro autor.

\section{REFERÊNCIAS}

ADAMS RP. 1995. Identification of Essential Oils by Gas Chromatography/Mass Spectroscopy. Carol Stream: Allured Publishing Corporation. 475p.

BEZERRA AME; NASCIMENTO JÚNIOR FT; LEAL FR; CARNEIRO JGM. 2006. Rendimento de biomassa, óleo essencial, teores de fósforo e potássio de chambá em resposta à adubação orgânica e mineral. Revista Ciência Agronômica 37: 124-129.

BLANK AF; ARRIGONI-BLANK MF; SILVA PA; TORRES MER; MENEZES HJA. 2003. Efeitos de composições de substratos na produção de mudas de quiôio (Ocimum gratissimum L.). Revista Ciência Agronômica 34: 105-108.
BLANK AF; SILVA PA; ARRIGONI-BLANK MF; SILVA-MANN R; BARRETO MCV. 2005. Influência da adubação orgânica e mineral no cultivo de manjericão cv. Genovese. Revista Ciência Agronômica 36: 175-180.

CAVALCANTI ESB; MORAIS SM; LIMA MAA; SANTANA EWP. 2004. Larvicidal activity of essential oils from Brazilian plants against Aedes aegypti L. Memórias do Instituto Oswaldo Cruz 99: 541-544.

CHAVES FCM; MING LC; EHLERT PAD. 2002. Influence of organic fertilization on leaves and essential oil production of Ocimum gratissimum L. Acta Horticulturae 576: 273-275.

COSTA FILHO LO; ENCARNAÇÃO CRF; OLIVEIRA AFM. 2006. Influência hídrica e térmica no crescimento e desenvolvimento de Ocimum gratissimum L. Revista Brasileira de Plantas Medicinais 8: 8-13.

FARIA TJ; FERREIRA RS; YASSUMOTO L; SOUZA JRP; ISHIKAWA NK; BARBOSA AM. 2006. Antifungal activity of essential oil isolated from Ocimum gratissimum $\mathrm{L}$. (eugenol chemotype) against phytopathogenic fungi. Brazilian Archives of Biology and Technology 49: 867-871.

JIROVETZ L; BUCHBAUER G; SHAFI MP; KANIAMPADY MM. 2003. Chemotaxonomical analysis of the essential oil aroma compounds of four different Ocimum species from southern India. European Food Research and Tecnology 217: 120-124.

LORENZI H; MATOS FJA. 2002. Plantas medicinais do Brasil: nativas e exóticas cultivadas. Nova Odessa: Instituto Plantarum. 512p.
MATOS SH; INNECCO R; CRUZ GF; EHLERT PAD. 2000. Determinação da altura de corte em alfavaca-cravo. Horticultura Brasileira 18: 998-999.

NAKAMURA CV; UEDA-NAKAMURA T; BANDO E; MELO AFN; CORTEZ DAG; DIAS FILHO BP. 1999. Antibacterial activity of Ocimum gratissimum L. essential oil. Memórias do Instituto Oswaldo Cruz 94: 675-678.

PEREIRA RS; SUMITA TC; FURLAN MR; JORGE AOC; UENO M. 2004. Atividade antibacteriana de óleos essenciais em cepas isoladas de infecção urinária. Revista de Saúde Pública 38: 326-328.

ROSSET M; ZAMARION VM; FACCIONE M; FARIA TJ; PINTO JP; BARBOSA AM; SOUZA JRP. 2005. Estudo químico da fração diclorometânica do extrato de Ocimum gratissimum L. Semina 26: 515520.

SANTOS MRA; INNECCO R. 2004. Adubação orgânica e altura de corte da erva-cidreira brasileira. Horticultura Brasileira 22: 182185.

SILVA MGV; CRAVEIRO AA; MATOS FJA; MACHADO MIL; ALENCAR JW. 1999. Chemical variation during daytime of constituents of the essential oil of Ocimum gratissimum leaves. Fitoterapia 70: 32-34.

SILVA PA; BLANK AF; ARRIGONI-BLANK MF; BARRETTO MCV. 2003. Efeitos da adubação orgânica e mineral na produção de biomassa e óleo essencial do capim-limão [Cymbopogon citratus (D.C.) Stapf]. Revista Ciência Agronômica 34: 92-96.

USIP LPE; OPARA KN; IBANGA ES; ATTING IA. 2006. Longitudinal evaluation of repellent activity of Ocimum gratissimum (Labiatae) volatile oil against Simulium damnosum. Memórias do Instituto Oswaldo Cruz 101: 201-205. 